EPJ manuscript No.

(will be inserted by the editor)

\title{
Thermal Casimir effect in ideal metal rectangular boxes
}

\author{
B. Geyer, G. L. Klimchitskaya ${ }^{a}$, and V. M. Mostepanenko ${ }^{b}$ \\ Center of Theoretical Studies and Institute for Theoretical Physics, Leipzig University, \\ Vor dem Hospitaltore 1, 100920, D-04009, Leipzig, Germany \\ Received: date / Revised version: date
}

\begin{abstract}
The thermal Casimir effect in ideal metal rectangular boxes is considered using the method of zeta functional regularization. The renormalization procedure is suggested which provides the finite expression for the Casimir free energy in any restricted quantization volume. This expression satisfies the classical limit at high temperature and leads to zero thermal Casimir force for systems with infinite characteristic dimensions. In the case of two parallel ideal metal planes the results, as derived previously using thermal quantum field theory in Matsubara formulation and other methods, are reproduced starting from the obtained expression. It is shown that for rectangular boxes the temperature-dependent contribution to the electromagnetic Casimir force can be both positive and negative depending on side lengths. The numerical computations of the scalar and electromagnetic Casimir free energy and force are performed for cubes.
\end{abstract}

\section{Introduction}

During the last few years there was an increasing interest to the Casimir effect [1, the physical phenomenon which is caused by the modification of the spectrum of zero-point and thermal oscillations in restricted quantization volumes and in spaces with nontrivial topology [2, 3. This effect has found prospective fundamental applications, e.g., for constraining predictions of modern unification theories beyond the standard model $4,5,6,7,8,9$ ' or in the braneworld cosmological scenaria [10,11,12]. Although much of recent research on the Casimir effect was concentrated in the experimental issues and material prop' erties [13, 14, 15], some of the principal field-theoretical aspects remain unsettled until the present time.

One of the most intriguing results is that the Casimir energy and force may change sign depending on geometry of the configuration and the type of boundary conditions. A dramatic example of this situation which has given rise to many discussions in the literature for several decades is a rectangular box with side lengths $a, b$ and $c$. Lukosz [16] noticed that the electromagnetic Casimir energy inside an ideal metal box may change sign depending on side sizes $a, b$ and $c$. The detailed investigation of the Casimir energy for fields of different spins, when it is positive or negative, inside a rectangular box as a function of box dimensions was performed by Mamayev and Trunov [17. 18. In particular, the analytical results for two- and threedimensional boxes at zero temperature were obtained by

\footnotetext{
${ }^{a}$ on leave from North-West Technical University, St.Petersburg, Russia

b on leave from Noncommercial Partnership "Scientific Instruments", Moscow, Russia
}

the repeated application of the Abel-Plana formula [2,13. Ambjørn and Wolfram [19] used the Epstein zeta function to calculate the Casimir energy for a scalar and electromagnetic field in hypercuboidal regions in $n$-dimensional space-time. The electromagnetic Casimir densities were investigated for a wedge [20] and for a wedge with a coaxial cylindrical shell 21. The problem of isolation of the divergent terms in the vacuum energy and their interpretation received the most attention. In recent years this problem was reformulated as a rectangular box or arbitrary shaped cavity divided into two sections by an ideal metal movable partition (piston) $[22,23,24,25,26,27$. It was shown that the Casimir force acting on the piston with Dirichlet boundary conditions attracts it to the nearest wall. Based on this, some doubts about the previously obtained results demonstrating Casimir repulsion in cubes have been raised. Both sets of results obtained for standard boxes and for boxes with a piston are, however, in mutual agreement. The attraction (or repulsion for a piston with Neumann boundary conditions [27]) of a piston to the nearest face of the box with any sides $a, b, c$ does not negate the Casimir repulsion for boxes without a piston that have some appropriate ratio of $a, b$ and $c$. The point is that the cases with an empty space outside the box and with another section of the larger box ouside the piston are quite different. In the first case the vacuum energy outside the box does not depend on $a, b$ and $c$ and there is no force acting on the box from the outside. Whereas in the second case there is an extra section of the larger box outside the piston which gives rise to the additional force acting on it.

Another intriguing problem to which this paper is devoted is the problem of the thermal Casimir effect in ideal 
metal rectangular boxes. The first calculations on this subject 19 resulted in a divergent free energy after removing the regularization. More recent results appear to be either infinite 28 or ambiguous 29]. Reference 30 reconsidered the derivation of the Casimir free energy for a massless scalar and electromagnetic field using zeta functional regularization. However, as shown below, the used formalism does not include all necessary subtractions. This led to unjustified conclusions on the behavior of the Casimir free energy as a function of temperature. Specifically, it is claimed that in rectangular cavities in the high temperature regime the leading term of the free energy is of order $\left(k_{B} T\right)^{4}$, where $k_{B}$ is the Boltzmann constant 30 . The terms of orders $\left(k_{B} T\right)^{3}$ and $\left(k_{B} T\right)^{2}$ are also obtained 30. The coefficients near such terms unavoidably depend of $a, b$ and $c$ and, thus, they contribute to the Casimir force.

In the present paper we consider the thermal Casimir effect in rectangular boxes starting from the general expression for the Casimir free energy in the framework of Matsubara quantum field theory at nonzero temperature. The thermal correction in this expression is renormalized in the same way as the zero-temperature contribution. We critically reconsider the known results from a unified point of view, separating clearly the empty space contribution in the volume of a body and other geometrical terms not only in the Casimir energy at zero temperature, but also in the temperature correction to it. Using the method of zeta functional regularization [31,32,33, the finite expression for the Casimir free energy associated with a volume $V$ is obtained. This expression is first applied to the familiar case of two plane parallel planes and the standard results 2, 3, 13,34 are reproduced. The Casimir force, as defined in this paper, goes to zero when the characteristic dimensions of the volume $V$ go to infinity. This is achieved by a subtraction of the free energy of the black body radiation within the volume $V$ and of two other geometrical contributions of quantum origin. Then we apply obtained general expression for the Casimir free energy to the cases of the massless scalar field with Dirichlet boundary conditions and the electromagnetic field in rectangular boxes. We show that the temperature-dependent contribution to the Casimir force at zero temperature can be both positive and negative (i.e., it leads to repulsion or attraction) depending on the box side lengths. This property is preserved for boxes with zero electromagnetic Casimir force at zero temperature. Numerical computations of the scalar and electromagnetic Casimir force as functions of side length and temperature for cubes are performed.

The paper is organized as follows. In Sect. 2 the general expression for the Casimir free energy is derived using the method of zeta functional regularization. Section 3 contains the application of this expression to the case of two plane parallel planes. In Sects. 4 and 5 the massless scalar and the electromagnetic fields, respectively, are considered in 3-dimensional rectangular boxes. Section 6 contains our conclusions and discussion. Throughout the paper the system of units is used where $c=\hbar=1$.

\section{The Casimir energy in Matsubara formulation and zeta functional regularization}

In the Matsubara formalism one uses an Euclidean field theory, considered as a continuation of the field theory in Minkowski space-time by a rotation of the time $t \rightarrow-\mathrm{i} \tau$. The Euclidean time $\tau$ is confined to the interval $\tau \in[0, \beta]$, where $\beta=1 /\left(k_{B} T\right)$ is the equivalent dimension corresponding to the inverse temperature. The bosonic fields must be periodic, $\varphi(\tau+\beta, \boldsymbol{r})=\varphi(\tau, \boldsymbol{r})$. In the limit of zero temperature one re-obtains the theory on the whole time axis.

In the Matsubara formalism, the partition function $Z$ has the following representation in terms of a functional integral,

$$
Z=C \int D \varphi e^{-S_{E}[\varphi]},
$$

where $S_{E}[\varphi]$ is the Euclidean action. It can be obtained from the corresponding one in Minkowski space-time by the replacement $S$ with i $S_{E}$. For example, for a scalar field with mass $m$ it is

$$
S_{E}[\phi]=\frac{1}{2} \int_{0}^{\beta} d \tau \int d \boldsymbol{r} \varphi K_{E} \varphi
$$

where

$$
K_{E}=\left(-\square_{E}+m^{2}\right)
$$

The Euclidean wave operator $\square_{E}=\partial^{2} / \partial \tau^{2}+\Delta$ is the continuation of the D'Alembert operator. In the functional integral (1), the field to be integrated over must fulfill the corresponding periodicity conditions.

In general, in the Matsubara formalism, the construction of the theory, to a large extent, goes in parallel to the zero temperature case. This is true for instance in the calculation of the functional integral. Since we consider free field theories, the functional integral is Gaussian and can be calculated directly. Using the infinite dimensional analogue to the standard finite dimensional relations, we obtain for the partition function from (11)

$$
Z=C\left(\operatorname{det} K_{E}\right)^{-1 / 2},
$$

where $C$ is an irrelevant constant which will be dropped below. Further, for the free energy we get

$$
\mathcal{F}=-\frac{1}{\beta} \ln Z=\frac{1}{2 \beta} \operatorname{Tr} \ln K_{E} .
$$

The trace in this expression is taken over the same space of fields to be integrated over in the functional integral (11).

Since we assume thermal equilibrium, it is always possible to separate the Euclidean time variable from the spatial variables. Assuming for the spatial part an eigenfunction expansion

$$
-\nabla^{2} \Phi_{J}(\boldsymbol{r})=\Lambda_{J} \Phi_{J}(\boldsymbol{r}), \quad \Lambda_{J} \equiv \omega_{J}^{2}-m^{2},
$$

where $J$ is a collective index, we obtain a basis in the space of fields $\varphi$,

$$
\Phi_{l, J}(\tau, \boldsymbol{r})=\frac{\mathrm{e}^{-\mathrm{i} \xi_{l} \tau}}{\sqrt{2 \pi}} \Phi_{J}(\boldsymbol{r}),
$$


with the so-called Matsubara frequencies

$$
\xi_{l}=2 \pi k_{B} T l, \quad l=0, \pm 1, \pm 2, \ldots .
$$

These functions are periodic in $\tau$ and these are eigenfunctions of $K_{E}$,

$$
K_{E} \Phi_{l, J}=\left(\xi_{l}^{2}+\Lambda_{J}+m^{2}\right) \Phi_{l, J} .
$$

As a consequence, the trace in the free energy becomes a sum over the logarithm of the eigenvalues,

$$
\mathcal{F}_{0}=\frac{1}{2} k_{B} T \sum_{l=-\infty}^{\infty} \sum_{J} \ln \left(\xi_{l}^{2}+\Lambda_{J}+m^{2}\right)
$$

Note that if the field is massless and all quantum numbers in the collective index $J$ are discrete, it is assumed in (10) that there are no physical states with $\Lambda_{J}=0$.

In (10), $\mathcal{F}_{0}$ is the free energy of an ensemble of states containing particles at the temperature $T$. In the special case of $T \rightarrow 0$, the time interval stretches over the whole axis and the sum over the Matsubara frequencies becomes the integral over the frequency $\xi$,

$$
k_{B} T \sum_{l=-\infty}^{\infty} f\left(\xi_{l}\right) \rightarrow \int_{-\infty}^{\infty} \frac{d \xi}{2 \pi} f(\xi)
$$

[here $f\left(\xi_{l}\right)$ is a function which must allow for an analytic continuation from discrete values to continuous ones]. In this way, $\mathcal{F}_{0}$ defined in (10) turns into the vacuum energy $E_{0}$.

The free energy, as given by (10), still contains ultraviolet divergences and one has to introduce a regularization. In zeta functional regularization, the free energy becomes

$$
\mathcal{F}_{0}(s)=-\frac{1}{2} \frac{\partial}{\partial s} \mu^{2 s} k_{B} T \sum_{l=-\infty}^{\infty} \sum_{J}\left(\xi_{l}^{2}+\Lambda_{J}+m^{2}\right)^{-s} .
$$

The regularization is removed for $s \rightarrow 0$ and $\mu$ is an arbitrary parameter with the dimension of mass. The separation of the ultraviolet divergences can be done quite easily because these are the same as at zero temperature. There are two ways to proceed. In the first method one has to apply the Abel-Plana formula 2,13 to the frequency sum in (12). This way has the advantage that it can also be applied to the case when there is an additional dependence on $l$, for example through the dielectric permittivity entering in the form of $\xi^{2} \rightarrow \varepsilon(\mathrm{i} \xi) \xi^{2}$. Another, to some extent easier, way is through the application of the Poisson summation formula 35. According to this formula, if $c(\alpha)$ is the Fourier transform of a function $b(x)$,

$$
c(\alpha)=\frac{1}{2 \pi} \int_{-\infty}^{\infty} b(x) \mathrm{e}^{-\mathrm{i} \alpha x} d x
$$

then it follows that

$$
\sum_{l=-\infty}^{\infty} b(l)=2 \pi \sum_{l=-\infty}^{\infty} c(2 \pi l)
$$

By putting

$$
b(x)=\mathrm{e}^{-z x^{2}}, \quad c(\alpha)=\frac{1}{2 \sqrt{\pi z}} \mathrm{e}^{-\frac{\alpha^{2}}{4 z}},
$$

we obtain from (14) the following equality:

$$
\sum_{l=-\infty}^{\infty} \mathrm{e}^{-z l^{2}}=\sqrt{\frac{\pi}{z}} \sum_{n=-\infty}^{\infty} \mathrm{e}^{-\pi^{2} n^{2} / z},
$$

where $\operatorname{Re} z>0$ is assumed. In order to use this equality we represent (12) as a parametric integral,

$\mathcal{F}_{0}(s)=-\frac{1}{2 \beta} \frac{\partial}{\partial s} \mu^{2 s} \int_{0}^{\infty} \frac{d t}{t} \frac{t^{s}}{\Gamma(s)} \sum_{l=-\infty}^{\infty} \sum_{J} \mathrm{e}^{-t\left(\xi_{l}^{2}+\Lambda_{J}+m^{2}\right)}$

and apply (16) with $z=\left(2 \pi k_{B} T\right)^{2} t=(2 \pi / \beta)^{2} t$. The result is

$$
\begin{gathered}
\mathcal{F}_{0}(s)=-\frac{1}{2} \frac{\partial}{\partial s} \mu^{2 s} \sum_{n=-\infty}^{\infty} \int_{0}^{\infty} \frac{d t}{t} \frac{t^{s}}{\Gamma(s) \sqrt{4 \pi t}} \\
\times \sum_{J} \mathrm{e}^{-\frac{n^{2} \beta^{2}}{4 t}-t\left(\Lambda_{J}+m^{2}\right)} .
\end{gathered}
$$

The $n$-dependent factor in the exponential provides convergence for the $t$-integration at $t \rightarrow 0$ for all terms in the sum over $n$ except for $n=0$. The latter is just the zero temperature contribution. This can be seen by applying (11) to the frequency sum in (17),

$$
k_{B} T \sum_{l=-\infty}^{\infty} \mathrm{e}^{-t \xi_{l}^{2}} \underset{T \rightarrow 0}{\rightarrow} \int_{-\infty}^{\infty} \frac{d \xi}{2 \pi} \mathrm{e}^{-t \xi^{2}}=\frac{1}{\sqrt{4 \pi t}} .
$$

As a consequence, we can split the free energy into the zero temperature part and the temperature dependent addition $\Delta_{T} \mathcal{F}_{0}$ as

$$
\mathcal{F}_{0}(s)=E_{0, \text { eff }}(s)+\Delta_{T} \mathcal{F}_{0}(s) .
$$

Here, the vacuum energy at zero temperature is

$$
E_{0, \mathrm{eff}}(s)=-\frac{1}{2} \frac{\partial}{\partial s} \mu^{2 s} \int_{-\infty}^{\infty} \frac{d \xi}{2 \pi} \sum_{J}\left(\xi^{2}+\Lambda_{J}+m^{2}\right)^{-s} .
$$

The nonrenormalized thermal correction is given by

$$
\begin{aligned}
\Delta_{T} \mathcal{F}_{0}(s)=- & \frac{\partial}{\partial s} \mu^{2 s} \sum_{n=1}^{\infty} \int_{0}^{\infty} \frac{d t}{t} \frac{t^{s}}{\Gamma(s) \sqrt{4 \pi t}} \\
& \times \sum_{J} \mathrm{e}^{-\frac{n^{2} \beta^{2}}{4 t}-t\left(\Lambda_{J}+m^{2}\right)} .
\end{aligned}
$$

Note that (20) has a transparent physical interpretation only for the temperature independent boundary conditions considered here.

The ultraviolet divergences are contained in $E_{0, \mathrm{eff}}(s)$ and can be dealt with in the standard way 2,13. This results in the replacement of $E_{0, \text { eff }}(s)$ with $E_{0}^{\text {ren }}$ in (20), where $E_{0}^{\text {ren }}$ is the finite renormalized Casimir energy at 
zero temperature associated with a volume $V$, which goes to zero when $V \rightarrow \infty$. In $\Delta_{T} \mathcal{F}_{0}(s)$ in (22), the integration over $t$ is convergent and we can remove the regularization, i.e., we can put $s=0$ using the equality

$$
\lim _{s \rightarrow 0} \frac{\partial}{\partial s} \frac{f(s)}{\Gamma(s)}=f(0),
$$

where $f(s)$ is any regular function at $s=0$. Following this, the integration over $t$ and the summation over $n$ can be carried out explicitly

$$
\Delta_{T} \mathcal{F}_{0}=k_{B} T \sum_{J} \ln \left(1-e^{-\beta \sqrt{\Lambda_{J}+m^{2}}}\right) .
$$

In this formula, we see the sum of the $T$-dependent contributions to the free energies of the individual degrees of freedom, or modes $\Lambda_{J}$, of the considered system. Taking into account $\Lambda_{J}+m^{2}=\omega_{J}^{2}$, the total free energy of all the oscillator modes appears to be

$$
\mathcal{F}_{0}=E_{0}^{\mathrm{ren}}+k_{B} T \sum_{J} \ln \left(1-\mathrm{e}^{-\beta \omega_{J}}\right),
$$

where the zero temperature contribution is already replaced with $E_{0}^{\text {ren }}$. For instance, if we take the volume $V$ to be that of empty space, the modes are plane waves, the index $J$ becomes the wave vector $\boldsymbol{k}$ and the sum over $J$ turns into the corresponding momentum integration with respect to $d \boldsymbol{k} /(2 \pi)^{3}$. As a result, from (24) we obtain the free energy density of the blackbody radiation

$$
f_{\mathrm{bb}}(T)=k_{B} T \int \frac{d \boldsymbol{k}}{(2 \pi)^{3}} \ln \left(1-e^{-\beta|\boldsymbol{k}|}\right)=-\frac{\pi^{2}\left(k_{B} T\right)^{4}}{90} .
$$

We note that (26) holds for a scalar field. For the electromagnetic field one would have to multiply by a factor of 2 . In fact, for the empty space $f_{\mathrm{bb}}$ defined in (26) is the complete free energy. This is because in this case the zero temperature part was the vacuum energy of empty space which was disregarded in the replacement of $E_{0 \text {,eff }}$ with $E_{0}^{\text {ren }}$. Using the thermodynamic connection between the energy at a temperature $T$ and the free energy

$$
U(T)=-T^{2} \frac{\partial}{\partial T} \frac{\mathcal{F}(T)}{T},
$$

it is evident that for $\mathcal{F}(T)=f_{\mathrm{bb}}^{\mathrm{em}}(T)=2 f_{\mathrm{bb}}(T)$ the respective energy density is in agreement with Planck's blackbody radiation density

$$
u=\frac{\pi^{2}\left(k_{B} T\right)^{4}}{15} .
$$

If we consider the free energy in a restricted volume $V$, then in accordance with (25), we have to keep the zero temperature part $E_{0}^{\text {ren }}$, where the empty space contribution has been dropped already. For the temperature dependent part, we have to note that we are interested in the change in energy which comes from the volume $V$. Therefore we need to subtract from the temperature dependent part $\Delta_{T} \mathcal{F}_{0}$ of the free energy, the corresponding amount related to empty space, i.e., the blackbody radiation density $f_{\mathrm{bb}}$ multiplied by the volume $V$. As a result, we come to the following expression for the renormalized free energy associated with a finite volume $V$,

$$
\mathcal{F}=E_{0}^{\mathrm{ren}}+\Delta_{T} \mathcal{F}_{0}-V f_{\mathrm{bb}},
$$

which can be considered as the relevant quantity for the Casimir effect at finite temperature. In the next section we show that this is the case for the configuration of two parallel ideal metal planes.

However, in general case (29) is not satisfactory and requires at least two additional subtractions in order to get the physical Casimir free energy. The point is that the asymptotic expression of the thermal correction, $\Delta_{T} \mathcal{F}_{0}$, in the limit of high temperatures (large separations) in addition to the leading term,

$$
-V \frac{\pi^{2}\left(k_{B} T\right)^{4}}{90(\hbar c)^{3}}
$$

contains two other similar terms

$$
\alpha_{1} \frac{\left(k_{B} T\right)^{3}}{(\hbar c)^{2}}+\alpha_{2} \frac{\left(k_{B} T\right)^{2}}{\hbar c},
$$

where $\alpha_{1}$ and $\alpha_{2}$ have dimensions of $\mathrm{cm}^{2}$ and $\mathrm{cm}$, respectively. They are expressed through the heat kernel coefficients $a_{1 / 2}$ and $a_{1}$, respectively [13. This was shown quite generally by Dowker and Kennedy [36]. We use usual units in (30) and (31) to underline the quantum character of all these terms. The coefficients $\alpha_{1}$ and $\alpha_{2}$, generally speaking, depend on geometrical parameters of the configuration (for two parallel planes $\alpha_{1}=\alpha_{2}=0$, but for a rectangular box $\alpha_{1}$ and $\alpha_{2}$ depend on the side sizes; see Sects. 4 and 5). Because of this, the terms of the form (31) in the free energy would lead to forces of quantum nature which do not vanish with increasing characteristic sizes of the body (the next expansion term in the high-temperature limit of the free energy has the form of $\alpha_{3} k_{B} T$ with a dimensionless coefficient $\alpha_{3}$; it is of classical origin and does not contribute to the Casimir force). Below we demonstrate that for rectangular boxes the geometrical structure of the coefficients $\alpha_{1}$ and $\alpha_{2}$ is just the same as in the two infinite terms subtracted from the zero-temperature Casimir energy to make it finite in the cutoff regularization 2,13. Thus, one can perform additional, finite, renormalization of the free energy resulting in

$$
\begin{aligned}
& \mathcal{F}^{\text {phys }}=E_{0}^{\text {ren }}+\Delta_{T} \mathcal{F}, \\
& \Delta_{T} \mathcal{F}=\Delta_{T} \mathcal{F}_{0}-V f_{\text {bb }}-\alpha_{1}\left(k_{B} T\right)^{3}-\alpha_{2}\left(k_{B} T\right)^{2} .
\end{aligned}
$$

Here, the quantity $\Delta_{T} \mathcal{F}$ is the physical thermal correction to the Casimir energy. The respective Casimir force obviously vanishes when the characteristic sizes of the volume $V$ along all three coordinate axes go to infinity.

Below we use (32) to investigate the thermal Casimir effect in configurations of two parallel ideal metal planes (Sect. 3) and rectangular boxes (Sects. 4 and 5). All other thermodynamic quantities like force and entropy can be 
also derived from this formula. As for configurations containing translational invariant directions like parallel planes or a cylinder, one should bear in mind that all quantities in (32) must be divided by corresponding parameters like the area of plates.

\section{The electromagnetic Casimir free energy between two parallel planes}

The Casimir free energy in the configuration of two parallel ideal metal planes can be easily obtained from the general equation (32). It is common knowledge that for two parallel planes the high-temperature asymptotic expansion of $\Delta_{T} \mathcal{F}_{0}$ does not contain terms presented in (31). Then (32) and (24) with $\alpha_{1}=\alpha_{2}=0$ represent the electromagnetic Casimir free energy per unit area in the form (prime adds a multiple $1 / 2$ to the term with $n=0$ )

$$
\begin{aligned}
& \mathcal{F}_{\mathrm{el}}^{\mathrm{phys}}(a, T)=-\frac{\pi^{2}}{720 a^{3}} \\
& +\frac{k_{B} T}{\pi} \int_{0}^{\infty} k_{\perp} d k_{\perp} \sum_{n=0}^{\infty} \ln \left(1-\mathrm{e}^{-\beta \omega_{k_{\perp}, n}}\right)+\frac{\pi^{2}\left(k_{B} T\right)^{4} a}{45},
\end{aligned}
$$

where

$$
\omega_{J}=\omega_{k_{\perp}, n}=\sqrt{k_{\perp}^{2}+\left(\frac{\pi n}{a}\right)^{2}} .
$$

By introducing the new variable

$$
z=\beta \omega_{k_{\perp}, n}=\frac{1}{k_{B} T} \sqrt{k_{\perp}^{2}+\left(\frac{\pi n}{a}\right)^{2}}
$$

and changing the order of summation and integration, we rearrange (33) as

$$
\begin{aligned}
& \mathcal{F}_{\mathrm{el}}^{\text {phys }}(a, T)=-\frac{\pi^{2}}{720 a^{3}} \\
& +\frac{\left(k_{B} T\right)^{3}}{\pi} \sum_{n=0}^{\infty} \int_{2 \pi n t}^{\infty} z d z \ln \left(1-\mathrm{e}^{-z}\right)+\frac{\pi^{2}}{720 a^{3} t^{4}},
\end{aligned}
$$

where $t \equiv T_{\text {eff }} / T$, and the effective temperature is defined in the usual units as $k_{B} T_{\text {eff }}=\hbar c /(2 a)$. The integral entering (36) can be evaluated using the series expansion

$$
\begin{gathered}
\int_{2 \pi n t}^{\infty} z d z \ln \left(1-\mathrm{e}^{-z}\right)=-\sum_{l=1}^{\infty} \frac{1}{l} \int_{2 \pi n t}^{\infty} z d z \mathrm{e}^{-l z} \\
=-\frac{1}{8 \pi a^{3}} \sum_{l=1}^{\infty} \frac{1}{(l t)^{3}}(1+2 \pi n l t) \mathrm{e}^{-2 \pi n l t}
\end{gathered}
$$

Substituting this into (36) and performing the summation in $n$, we obtain

$$
\begin{gathered}
\mathcal{F}_{\mathrm{el}}^{\text {phys }}(a, T)=-\frac{\pi^{2}}{720 a^{3}}\left\{1+\frac{45}{\pi^{3}} \sum_{l=1}^{\infty}\left[\frac{\operatorname{coth}(\pi l t)}{t^{3} l^{3}}\right.\right. \\
\left.\left.+\frac{\pi}{t^{2} l^{2} \sinh ^{2}(\pi t l)}\right]-\frac{1}{t^{4}}\right\} .
\end{gathered}
$$

This is a well known result $2,3,13,34,37$, and the last term on the right-hand side of (38) originates from the subtracted contribution of blackbody radiation. At low temperature $T \ll T_{\text {eff }}($ (38) $)$ leads to [2,3, 13, 34, 37]

$\mathcal{F}_{\mathrm{el}}^{\text {phys }}(a, T)=-\frac{\pi^{2}}{720 a^{3}}\left[1+\frac{45 \zeta(3)}{\pi^{3}}\left(\frac{T}{T_{\text {eff }}}\right)^{3}-\left(\frac{T}{T_{\mathrm{eff}}}\right)^{4}\right]$,

where $\zeta(z)$ is the Riemann zeta function, and exponentially small terms are omitted. We emphasize that the quantity $1 /\left(a T_{\text {eff }}\right)^{3}$ does not depend on $a$. Because of this, only the last term on the right-hand side of (39), originating from the subtraction of the blackbody radiation in (32), contributes to the measurable quantity, the Casimir pressure following from (39)

$$
P_{\mathrm{el}}(a, T)=-\frac{\partial \mathcal{F}_{\mathrm{el}}^{\mathrm{phys}}(a, T)}{\partial a}=-\frac{\pi^{2}}{240 a^{4}}\left[1+\frac{1}{3}\left(\frac{T}{T_{\mathrm{eff}}}\right)^{4}\right] .
$$

Thus, if this subtraction would not be done in (32) and (33), only the exponentially small thermal corrections of order $\left(T / T_{\text {eff }}\right) \exp \left(-2 \pi T_{\text {eff }} / T\right)$ were added to unity on the right-hand side of (40).

In a similar way, at high temperature $T \gg T_{\text {eff }}$ (38) results in $[2,3,13,34,37$.

$$
\mathcal{F}_{\mathrm{el}}^{\text {phys }}(a, T)=-\frac{k_{B} T}{8 \pi a^{2}} \zeta(3), \quad P_{\mathrm{el}}(a, T)=-\frac{k_{B} T}{4 \pi a^{3}} \zeta(3) .
$$

This is the commonly known classical limit where the result does not depend on the Planck constant [38,39]. It is obtained because at high temperature the free energy of the blackbody radiation is canceled by the other terms contained in (38). If the subtraction of the blackbody radiation in (32) were not done, the term of order $\left(k_{B} T\right)^{4} /(c \hbar)^{3}$ (in usual units) would be dominating at high temperature (or large separations) in contradiction with the classical limit.

\section{Scalar Casimir effect in rectangular boxes}

After the above demonstration that the general equation (32) leads to the familiar result in the case of two parallel planes, we apply it to calculate the thermal Casimir effect in rectangular boxes with sides $a, b$ and $c$. We start with the case of a massless scalar field with Dirichlet boundary conditions. Then (32), (24) can be written in the form

$$
\begin{aligned}
\mathcal{F}^{\text {phys }}(a, b, c, T)=E_{0}^{\text {ren }}(a, b, c) \\
\quad+k_{B} T \sum_{n, l, p=1}^{\infty} \ln \left(1-\mathrm{e}^{-\beta \omega_{n l p}}\right) \\
+\frac{\pi^{2}\left(k_{B} T\right)^{4}}{90} a b c-\alpha_{1}\left(k_{B} T\right)^{3}-\alpha_{2}\left(k_{B} T\right)^{2},
\end{aligned}
$$

where

$$
\omega_{n l p}^{2}=\pi^{2}\left(\frac{n^{2}}{a^{2}}+\frac{l^{2}}{b^{2}}+\frac{p^{2}}{c^{2}}\right), \quad n, l, p=1,2,3, \ldots
$$


and the Casimir energy at zero temperature is given by 40,41

$$
\begin{gathered}
E_{0}^{\mathrm{ren}}(a, b, c)=-\frac{\pi^{2} b c}{1440 a^{3}}+\frac{\zeta(3)(b+c)}{32 \pi a^{2}}-\frac{\pi}{96 a} \\
-\frac{\pi}{2 a}\left[G\left(\frac{b}{a}\right)+G\left(\frac{c}{a}\right)\right]-\frac{1}{a} R\left(\frac{b}{a}, \frac{c}{a}\right)
\end{gathered}
$$

Here the following notations are introduced:

$$
\begin{aligned}
& G(z)=-\frac{1}{2 \pi} \sum_{n, l=1}^{\infty} \frac{n}{l} K_{1}(2 \pi n l z) \\
& R\left(z_{1}, z_{2}\right) \equiv \frac{z_{1} z_{2}}{8} \sum_{l, p=-\infty}^{\infty}\left(1-\delta_{l 0} \delta_{p 0}\right) \\
& \quad \times \sum_{j=1}^{\infty}\left(\frac{j}{\sqrt{l^{2} z_{1}^{2}+p^{2} z_{2}^{2}}}\right)^{3 / 2} K_{3 / 2}\left(2 \pi j \sqrt{l^{2} z_{1}^{2}+p^{2} z_{2}^{2}}\right)
\end{aligned}
$$

and $K_{n}(z)$ are Bessel functions of imaginary argument.

Equation (42) presents the finite renormalized value of the Casimir free energy for a scalar field with Dirichlet boundary conditions in a box with sides $a, b$ and $c$ valid at any temperature. However, the values of $\alpha_{1}$ and $\alpha_{2}$ remain unknown. To determine them we should find the asymptotic expression for the nonrenormalized thermal correction $\Delta_{T} \mathcal{F}_{0}$ at high temperatures (large separations). To do this, we identically rearrange $\Delta_{T} \mathcal{F}_{0}$ to the form

$$
\begin{aligned}
& \Delta_{T} \mathcal{F}_{0}(a, b, c, T)=k_{B} T X\left(\beta_{a}, \beta_{b}, \beta_{c}\right), \\
& X\left(\beta_{a}, \beta_{b}, \beta_{c}\right) \equiv \sum_{n, l, p=1}^{\infty} \ln \left(1-\mathrm{e}^{-\sqrt{\beta_{a}^{2} n^{2}+\beta_{b}^{2} l^{2}+\beta_{c}^{2} p^{2}}}\right),
\end{aligned}
$$

where

$$
\beta_{a}=\frac{\pi \beta}{a}, \quad \beta_{b}=\frac{\pi \beta}{b}, \quad \beta_{c}=\frac{\pi \beta}{c} .
$$

Note that the quantity $X$ does not depend on $a, b, c$ and $T$ separately, but only through the products $a T, b T$ and $c T$. Below we find the asymptotic expression of $X$ under the conditions $\beta_{a}, \beta_{b}, \beta_{c} \ll 1$. This can be done by the repeated application of the Abel-Plana formula [2,13]

$$
\sum_{n=1}^{\infty} f(n)=-\frac{1}{2} f(0)+\int_{0}^{\infty} f(t) d t+\int_{0}^{\infty} d t \frac{f(\mathrm{i} t)-f(-\mathrm{i} t)}{\mathrm{e}^{2 \pi t}-1} .
$$

First we put

$$
f(n)=\sum_{l, p=1}^{\infty} \ln \left(1-\mathrm{e}^{-\sqrt{\beta_{a}^{2} n^{2}+\beta_{b}^{2} l^{2}+\beta_{c}^{2} p^{2}}}\right) .
$$

Then the application of (48) leads to

$$
\begin{aligned}
& X\left(\beta_{a}, \beta_{b}, \beta_{c}\right)=-\frac{1}{2} \sum_{l, p=1}^{\infty} \ln \left(1-\mathrm{e}^{-\sqrt{\beta_{b}^{2} l^{2}+\beta_{c}^{2} p^{2}}}\right) \\
& +\int_{0}^{\infty} d t \sum_{l, p=1}^{\infty} \ln \left(1-\mathrm{e}^{-\sqrt{\beta_{a}^{2} t^{2}+\beta_{b}^{2} l^{2}+\beta_{c}^{2} p^{2}}}\right) \\
& +O\left(\ln \beta_{a}, \ln \beta_{b}, \ln \beta_{c}\right) .
\end{aligned}
$$

Here, it is taken into account that the last term on the right-hand side of (48) with $f$ defined in (49) is of order $\ln \beta_{a}, \ln \beta_{b}$ and $\ln \beta_{c}$.

Applying the Abel-Plana formula to each of the sums in (50), we get

$$
\begin{aligned}
& X\left(\beta_{a}, \beta_{b}, \beta_{c}\right)=\frac{1}{4} \sum_{p=1}^{\infty} \ln \left(1-\mathrm{e}^{-\beta_{c} p}\right) \\
& -\frac{1}{2}\left(\frac{1}{\beta_{a}}+\frac{1}{\beta_{b}}\right) \int_{0}^{\infty} d y \sum_{p=1}^{\infty} \ln \left(1-\mathrm{e}^{-\sqrt{y^{2}+\beta_{c}^{2} p^{2}}}\right) \\
& +\frac{1}{\beta_{a} \beta_{b}} \int_{0}^{\infty} d y \int_{0}^{\infty} d v \sum_{p=1}^{\infty} \ln \left(1-\mathrm{e}^{-\sqrt{y^{2}+v^{2}+\beta_{c}^{2} p^{2}}}\right) \\
& +O\left(\ln \beta_{a}, \ln \beta_{b}, \ln \beta_{c}\right) .
\end{aligned}
$$

Bearing in mind that

$$
\sum_{p=1}^{\infty} \ln \left(1-\mathrm{e}^{-\beta_{c} p}\right)=-\frac{\pi^{2}}{6 \beta_{c}}+O\left(\ln \beta_{c}\right)
$$

and applying the Abel-Plana formula to the remaining two sums, the following result is obtained:

$$
\begin{aligned}
X & \left(\beta_{a}, \beta_{b}, \beta_{c}\right)=-\frac{\pi^{2}}{24 \beta_{c}} \\
+ & \frac{1}{4}\left(\frac{1}{\beta_{a}}+\frac{1}{\beta_{b}}\right) \int_{0}^{\infty} d y \ln \left(1-\mathrm{e}^{-y}\right) \\
- & \frac{1}{2}\left(\frac{1}{\beta_{a} \beta_{c}}+\frac{1}{\beta_{b} \beta_{c}}+\frac{1}{\beta_{a} \beta_{b}}\right) \\
& \times \int_{0}^{\infty} d y \int_{0}^{\infty} d v \ln \left(1-\mathrm{e}^{-\sqrt{y^{2}+v^{2}}}\right) \\
+ & \frac{1}{\beta_{a} \beta_{b} \beta_{c}} \int_{0}^{\infty} d y \int_{0}^{\infty} d v \int_{0}^{\infty} d w \ln \left(1-\mathrm{e}^{-\sqrt{y^{2}+v^{2}+w^{2}}}\right) \\
+ & O\left(\ln \beta_{a}, \ln \beta_{b}, \ln \beta_{c}\right) .
\end{aligned}
$$

Calculating all integrals and using (46) and notations (47) we arrive at the asymptotic expression for the nonrenormalized thermal correction at high temperatures (large separations)

$$
\begin{aligned}
& \Delta_{T} \mathcal{F}_{0}(a, b, c, T)=-\frac{\pi}{24}\left(k_{B} T\right)^{2}(a+b+c) \\
& +\frac{\zeta(3)}{4 \pi}(a c+b c+a b)\left(k_{B} T\right)^{3}-\frac{\pi^{2}}{90}\left(k_{B} T\right)^{4} a b c \\
& +O\left(k_{B} T \ln \beta_{a}, k_{B} T \ln \beta_{b}, k_{B} T \ln \beta_{c}\right) .
\end{aligned}
$$

Thus, we have demonstrated that at high temperatures (large separations) the nonrenormalized thermal correction really contains the terms of form (30) and (31). From the comparison of (54) with (31), it follows

$$
\alpha_{1}=\frac{\zeta(3)}{4 \pi}(a c+b c+a b), \quad \alpha_{2}=-\frac{\pi}{24}(a+b+c),
$$

i.e., $\alpha_{1}$ is proportional to the total area of box surface, and $\alpha_{2}$ to the sum of the sides. These geometrical objects (together with a box volume) are usually renormalized when replacing $E_{0, \text { eff with } E_{0}^{\text {ren }}[2,13,17,18}$. Thus the 
subtraction of the last three terms on the right-hand side of (42) can be interpreted as an additional finite renormalization giving a physically meaningful temperaturedependent contribution to the Casimir energy.

As mentioned in Sect. 2, $\alpha_{1}$ and $\alpha_{2}$ can be expressed in terms of the heat kernel coefficients. Thus, keeping in mind that the heat kernel coefficient $a_{1 / 2}=-\sqrt{\pi} S / 2$ [4], where $S$ is the area of box surface, and comparing (54) with the general asymptotic expression for the free energy [36, one arrives at

$$
\alpha_{1}=-\frac{\zeta(3)}{4 \pi^{3 / 2}} a_{1 / 2}=\frac{\zeta(3)}{8 \pi} S
$$

in agreement with (55). Similar comparison of the general expression [36] with (54) leads to $\alpha_{2}=-a_{1} / 24$. The heat kernel coefficient $a_{1}$ can be calculated from the known expression for the heat kernel coefficient of an angle $\theta$ [4].

$$
c_{1}(\theta)=\frac{\pi^{2}-\theta^{2}}{6 \theta}
$$

In the case of rectangular box $\theta=\pi / 2$ and $c_{1}=\pi / 4$. In the 3 -dimensional case this should be multiplied by the length of all sides leading to

$$
a_{1}=4 c_{1}(a+b+c)=\pi(a+b+c) .
$$

From this, the expression for $\alpha_{2}$ in (55) is reobtained.

The Casimir force acting between the opposite faces of the box is obtained from (42) and (55)

$$
\begin{aligned}
& F_{x}(a, b, c, T)=-\frac{\partial \mathcal{F}^{\mathrm{phys}}(a, b, c, T)}{\partial a}=F_{x}(a, b, c) \\
& +\frac{\pi^{2}}{a^{3}} \sum_{n, l, p=1}^{\infty} \frac{n^{2}}{\omega_{n l p}\left(\mathrm{e}^{\beta \omega_{n l p}}-1\right)}-\frac{\pi^{2}\left(k_{B} T\right)^{4}}{90} b c \\
& +\frac{\zeta(3)}{4 \pi}\left(k_{B} T\right)^{3}(b+c)-\frac{\pi}{24}\left(k_{B} T\right)^{2} .
\end{aligned}
$$

It is well known [13, 41, that the scalar Casimir energy $E_{0}^{\mathrm{ren}}(a, b, c)$ is negative and the respective force $F_{x}(a, b, c)=$ $-\partial E(a, b, c) / \partial a$ is attractive for any ratio of $a, b$ and $c$. Because of this, we restrict ourselves to the consideration of a cube $a=b=c$.

In the limit of low temperature $T \ll T_{\text {eff }}$ the leading terms in (42) are

$$
\begin{gathered}
\mathcal{F}^{\text {phys }}(a, T)=E_{0}^{\text {ren }}(a)-k_{B} T \exp \left(-\frac{\pi \sqrt{3}}{a k_{B} T}\right) \\
+\frac{\pi^{2}\left(k_{B} T\right)^{4}}{90} a^{3}-\frac{3 \zeta(3)}{4 \pi}\left(k_{B} T\right)^{3} a^{2}+\frac{\pi}{24}\left(k_{B} T\right)^{2} a
\end{gathered}
$$

where the scalar Casimir energy for a cube can be calculated numerically using (44) and (45) with the result

$$
E_{0}^{\mathrm{ren}}(a)=-\frac{0.0102}{a} \text {. }
$$

At arbitrary temperature it is convenient to represent both the free energy and force in terms of the dimensionless variable $t$

$$
\mathcal{F}^{\text {phys }}(a, T)=E_{0}^{\text {ren }}(a)
$$

$$
\begin{aligned}
& +\frac{1}{2 a t} \sum_{n, l, p=1}^{\infty} \ln \left(1-\mathrm{e}^{-2 \pi t \sqrt{n^{2}+l^{2}+p^{2}}}\right) \\
& +\frac{\pi^{2}}{1440 a t^{4}}-\frac{3 \zeta(3)}{32 \pi} \frac{1}{a t^{3}}+\frac{\pi}{32} \frac{1}{a t^{2}}, \\
& F_{x}(a, T)=F_{x}(a) \\
& +\frac{\pi}{a^{2}} \sum_{n, l, p=1}^{\infty} \frac{n^{2}}{\sqrt{n^{2}+l^{2}+p^{2}}} \frac{1}{\mathrm{e}^{2 \pi t \sqrt{n^{2}+l^{2}+p^{2}}}-1} \\
& -\frac{\pi^{2}}{1440 a^{2} t^{4}}+\frac{\zeta(3)}{16 \pi} \frac{1}{a^{2} t^{3}}-\frac{\pi}{96} \frac{1}{a^{2} t^{2}},
\end{aligned}
$$

where the force at zero temperature in accordance with (61) is given by

$$
F_{x}(a)=-\frac{0.0102}{3 a^{2}}
$$

In Fig. 1(a) we plot the scalar Casimir free energy in a cube from (62) as a function of the side length $a$ at $T=300 \mathrm{~K}$ (solid line). As is seen from this figure, the free energy increases monotonously with the increase of $a$. At large separations (not shown in the figure) it approaches to a constant. In the same figure the Casimir energy $E_{0}^{\text {ren }}=$ $E_{0}^{\text {ren }}(a)$ at zero temperature is shown by the dashed line. In Fig. 1(b) the scalar Casimir free energy is plotted as a function of temperature in the cube with $a=2 \mu \mathrm{m}$. It is seen that at large temperatures $\mathcal{F}^{\text {phys }}$ is proportional to the temperature in accordance with the classical limit.

The magnitude of the scalar Casimir force from (62) (in a logarithmic scale) as a function of $a$ at $T=300 \mathrm{~K}$ is shown in Fig. 2(a) by the solid line. The force is attractive for cubes with any side length and its magnitude goes to zero with the increase of $a$. In the same figure the dashed line shows the magnitude of the Casimir force (in a logarithmic scale) acting on the opposite faces of the cube at $T=0$. Fig 2(b) shows the Casimir force as a function of temperature for the cube with $a=2 \mu \mathrm{m}$. It is seen that for both negative and positive values of the free energy the respective Casimir force is attractive.

\section{Electromagnetic Casimir effect in rectangular boxes}

Now we consider the electromagnetic thermal Casimir effect in a rectangular box with the side lengths $a, b$ and $c$. For the electromagnetic field the renormalized free energy (32), (24) is specified as

$$
\begin{aligned}
& \mathcal{F}_{\mathrm{el}}^{\mathrm{phys}}(a, b, c, T)=E_{0, \mathrm{el}}^{\mathrm{ren}}(a, b, c) \\
& +k_{B} T\left[\sum_{l, p=1}^{\infty} \ln \left(1-\mathrm{e}^{-\beta \omega_{l p}}\right)+\sum_{n, l=1}^{\infty} \ln \left(1-\mathrm{e}^{-\beta \omega_{n l}}\right)\right. \\
& \left.+\sum_{n, p=1}^{\infty} \ln \left(1-\mathrm{e}^{-\beta \omega_{n p}}\right)+2 \sum_{n, l, p=1}^{\infty} \ln \left(1-\mathrm{e}^{-\beta \omega_{n l p}}\right)\right]
\end{aligned}
$$




$$
+\frac{\pi^{2}\left(k_{B} T\right)^{4}}{45} a b c-\alpha_{1}^{\mathrm{el}}\left(k_{B} T\right)^{3}-\alpha_{2}^{\mathrm{el}}\left(k_{B} T\right)^{2} .
$$

Here, $\omega_{n l p}$ is defined in (43), $\omega_{n l}=\omega_{n l 0}$, and $\alpha_{1}^{\mathrm{el}}, \alpha_{2}^{\mathrm{el}}$ have to be determined. The electromagnetic Casimir energy at $T=0, E_{0}^{\mathrm{ren}}(a, b, c)$, is given by

$$
\begin{gathered}
E_{0, \mathrm{el}}^{\mathrm{ren}}(a, b, c)=-\frac{\pi^{2} b c}{720 a^{3}}-\frac{\zeta(3) c}{16 \pi b^{2}}+\frac{\pi}{48}\left(\frac{1}{a}+\frac{1}{b}\right) \\
+\frac{\pi}{b} G\left(\frac{c}{b}\right)-\frac{2}{a} R\left(\frac{b}{a}, \frac{c}{a}\right),
\end{gathered}
$$

where the functions $G$ and $R$ are defined in (45).

In order to find the coefficients $\alpha_{1}^{\mathrm{el}}$ and $\alpha_{2}^{\mathrm{el}}$, one should determine the asymptotic behavior of $\Delta_{T} \mathcal{F}_{0, \text { el }}$ in the limit of high temperatures (large separations). In the electromagnetic case the nonrenormalized thermal correction can be identically rearranged to the form

$$
\begin{aligned}
& \Delta_{T} \mathcal{F}_{0, \mathrm{el}}(a, b, c, T)=k_{B} T Y\left(\beta_{a}, \beta_{b}, \beta_{c}\right), \\
& Y\left(\beta_{a}, \beta_{b}, \beta_{c}\right)=2 X\left(\beta_{a}, \beta_{b}, \beta_{c}\right) \\
& \quad+\sum_{l, p=1}^{\infty} \ln \left(1-\mathrm{e}^{-\sqrt{\beta_{b}^{2} l^{2}+\beta_{c}^{2} p^{2}}}\right) \\
& \quad+\sum_{n, l=1}^{\infty} \ln \left(1-\mathrm{e}^{-\sqrt{\beta_{a}^{2} n^{2}+\beta_{b}^{2} l^{2}}}\right) \\
& \quad+\sum_{n, p=1}^{\infty} \ln \left(1-\mathrm{e}^{-\sqrt{\beta_{a}^{2} n^{2}+\beta_{c}^{2} p^{2}}}\right),
\end{aligned}
$$

where the asymptotic behavior of $X\left(\beta_{a}, \beta_{b}, \beta_{c}\right)$ at small $\beta_{a}, \beta_{b}$ and $\beta_{c}$ was already determined in Sect. 4. Taking into account (46), (47) and (54), it is given by

$$
\begin{gathered}
X\left(\beta_{a}, \beta_{b}, \beta_{c}\right)=-\frac{\pi^{2}}{24}\left(\frac{1}{\beta_{a}}+\frac{1}{\beta_{b}}+\frac{1}{\beta_{c}}\right) \\
+\frac{\pi \zeta(3)}{4}\left(\frac{1}{\beta_{a} \beta_{b}}+\frac{1}{\beta_{a} \beta_{c}}+\frac{1}{\beta_{b} \beta_{c}}\right) \\
-\frac{\pi^{5}}{90} \frac{1}{\beta_{a} \beta_{b} \beta_{c}}+O\left(\ln \beta_{a}, \ln \beta_{b}, \ln \beta_{c}\right) .
\end{gathered}
$$

The asymptotic behavior of $Y\left(\beta_{a}, \beta_{b}, \beta_{c}\right)$ at small $\beta_{a}, \beta_{b}, \beta_{c}$ is obtained in perfect analogy with the case of the scalar field by the repeated application of the Abel-Plana formula (48) to the remaining three summations in (66). The result obtained with account of (67) is

$$
\begin{gathered}
Y\left(\beta_{a}, \beta_{b}, \beta_{c}\right)=\frac{\pi^{2}}{12}\left(\frac{1}{\beta_{a}}+\frac{1}{\beta_{b}}+\frac{1}{\beta_{c}}\right) \\
-\frac{\pi^{5}}{45} \frac{1}{\beta_{a} \beta_{b} \beta_{c}}+O\left(\ln \beta_{a}, \ln \beta_{b}, \ln \beta_{c}\right) .
\end{gathered}
$$

Substituting this into (66) and using notations (47), one obtains the asymptotic expression for the thermal correction at high temperatures (large separations)

$$
\begin{aligned}
& \Delta_{T} \mathcal{F}_{0, \mathrm{el}}(a, b, c, T)=\frac{\pi}{12}\left(k_{B} T\right)^{2}(a+b+c) \\
& -\frac{\pi^{2}}{45}\left(k_{B} T\right)^{4} a b c+O\left(k_{B} T \ln \beta_{a}, k_{B} T \ln \beta_{b}, k_{B} T \ln \beta_{c}\right) .
\end{aligned}
$$

It is notable that (69) does not contain a contribution proportional to the surface area of the box [compare with (54) for the scalar field]. In the electromagnetic case such a contribution is absent also in the divergent Casimir energy of the box $E_{0, \mathrm{el}}$ at zero temperature [2,13, 17, 18]. Thus, from (69) and (31) one arrives at

$$
\alpha_{1}^{\mathrm{el}}=0, \quad \alpha_{2}^{\mathrm{el}}=\frac{\pi}{12}(a+b+c) .
$$

The Casimir force acting between the opposite faces of a box is obtained as the negative derivative of (64) with respect to $a$,

$$
\begin{aligned}
& F_{x, \mathrm{el}}(a, b, c, T)=F_{x, \mathrm{el}}(a, b, c)+\frac{\pi^{2}}{a^{3}}\left[\sum_{n, l=1}^{\infty} \frac{n^{2}}{\omega_{n l}\left(\mathrm{e}^{\beta \omega_{n l}}-1\right)}\right. \\
& \left.+\sum_{n, p=1}^{\infty} \frac{n^{2}}{\omega_{n p}\left(\mathrm{e}^{\beta \omega_{n p}}-1\right)}+2 \sum_{n, l, p=1}^{\infty} \frac{n^{2}}{\omega_{n l p}\left(\mathrm{e}^{\beta \omega_{n l p}}-1\right)}\right] \\
& -\frac{\pi^{2}\left(k_{B} T\right)^{4}}{45} b c+\frac{\pi}{12}\left(k_{B} T\right)^{2} .
\end{aligned}
$$

It has been known that the electromagnetic Casimir energy inside a box at $T=0, E_{0, \mathrm{el}}^{\mathrm{ren}}(a, b, c)$, can be both positive and negative and the Casimir force, $F_{x, \mathrm{el}}(a, b, c)=$ $-\partial E_{0, \mathrm{el}}^{\mathrm{ren}}(a, b, c) / \partial a$, can be both attractive and repulsive depending on the ratio of the sides $a, b$ and $c$ [2,13]. Here we consider in more detail the thermal electromagnetic Casimir effect for a cube $a=b=c$ where the electromagnetic Casimir energy at zero temperature computed by (65) is positive,

$$
E_{0, \mathrm{el}}^{\mathrm{ren}}(a)=\frac{0.09166}{a}>0,
$$

and the force (71) is repulsive.

For a cube the electromagnetic Casimir free energy (64) and force (71) are given by

$$
\begin{aligned}
& \mathcal{F}_{\mathrm{el}}^{\text {phys }}(a, T)=E_{0, \mathrm{el}}^{\mathrm{ren}}(a)+\frac{3}{2 a t} \sum_{n, l=1}^{\infty} \ln \left(1-\mathrm{e}^{-2 \pi t \sqrt{n^{2}+l^{2}}}\right) \\
& +\frac{1}{a t} \sum_{n, l, p=1}^{\infty} \ln \left(1-\mathrm{e}^{-2 \pi t \sqrt{n^{2}+l^{2}+p^{2}}}\right)+\frac{\pi^{2}}{720 a t^{4}}-\frac{\pi}{16 a t^{2}} \\
& F_{x, \mathrm{el}}(a, T)=F_{x, \mathrm{el}}(a) \\
& +\frac{2 \pi}{a^{2}}\left[\sum_{n, l=1}^{\infty} \frac{n^{2}}{\sqrt{n^{2}+l^{2}}} \frac{1}{\mathrm{e}^{2 \pi t \sqrt{n^{2}+l^{2}}}-1}\right. \\
& \left.+\sum_{n, l, p=1}^{\infty} \frac{n^{2}}{\sqrt{n^{2}+l^{2}+p^{2}}} \frac{1}{\mathrm{e}^{2 \pi t \sqrt{n^{2}+l^{2}+p^{2}}}-1}\right] \\
& \quad-\frac{\pi^{2}}{720 a^{2} t^{4}}+\frac{\pi}{48 a^{2} t^{2}},
\end{aligned}
$$

where the force at $T=0$ is

$$
F_{x, \mathrm{el}}(a)=\frac{0.09166}{3 a^{2}}
$$


In Fig. 3(a) we plot the electromagnetic Casimir free energy in a cube as a function of $a$ at $T=300 \mathrm{~K}$ (solid line). In the same figure the Casimir energy at $T=0$ is shown by the dashed line. As is seen in this figure, the electromagnetic Casimir free energy decreases with the increase of separation. Similar to the scalar case, at large separations $\mathcal{F}_{\text {el }}^{\text {phys }}$ approaches to a constant. In Fig. 3(b) the electromagnetic Casimir free energy is shown as a function of temperature for a cube with $a=2 \mu \mathrm{m}$. The free energy decreases with the increase of $T$. At high temperatures $\mathcal{F}_{\mathrm{el}}^{\text {phys }}$ demonstrates the classical limit. The respective thermal electromagnetic Casimir force at $T=300 \mathrm{~K}$, as a function of $a$, is shown in Fig. 4(a) by the solid line. It is positive (i.e., repulsive) for cubes with any side lengths. Thus, thermal effects for cubes in the electromagnetic case increase the strength of the Casimir repulsion. The dashed line in Fig. 4(a) shows the electromagnetic Casimir force at $T=0$ as a function of $a$. This force is given by (74), i.e., it is always repulsive. Fig. 4(b) demonstrates the electromagnetic Casimir force in a cube of side length $a=2 \mu \mathrm{m}$ as a function of temperature. It is seen that the force increases with increasing temperature.

Note that the obtained results differ from those found in [30], where the terms of order $\left(k_{B} T\right)^{4}$ and of lower orders in the Casimir free energy were obtained in the hightemperature regime. Also, the Casimir free energy in 30. both for the scalar and electromagnetic field is always a decreasing function of temperature on the opposite to our result in Fig. 1(b). This is explained by the fact that in [30] the subtractions of the contributions from the blackbody radiation and of the terms proportional to the box surface area and to the sum of its sides were not made.

The above equations (42), (59), (64) and (71) can be used to compute the scalar and electromagnetic free energy and force for boxes with arbitrary side lengths $a, b$ and $c$. Specifically it follows that the temperature-dependent contribution to the electromagnetic Casimir force [which is obtained as $-\partial \Delta_{T} \mathcal{F} / \partial a$ from the physical thermal correction defined in (32)] can be both positive and negative depending on the side lengths $a, b$ and $c$. On the one hand, as shown above (see Fig. 4), for a cube $a \times a \times a$ the temperature-dependent contribution to the Casimir force is positive and computations show that this is preserved for any box $a \times b \times b$ with $a>b$. On the other hand, for boxes with $b=c=10 \mu \mathrm{m}$ and $a_{1}=2.942 \mu \mathrm{m}$ or $a_{2}=34.29 \mu \mathrm{m}$ the Casimir energy at $T=0$ is equal to zero [2,13. Computations using (71) show that for the box $a_{1} \times b \times b$ the temperature-dependent contribution to the force is negative, whereas for the box $a_{2} \times b \times b$ it is positive.

The thermal correction to the Casimir energy and force acting on a piston were investigated 24] for the scalar field with Dirichlet or Neumann boundary conditions using (25). The electromagnetic Casimir free energy and force acting on a piston were found in the case of ideal metal rectangular boxes and cavities with general cross section 24. In the limit of low temperatures the thermal correction to the Casimir force on a piston was shown to be exponentially small. In the limit of medium tempera- ture 24] obtains the term of order $\left(k_{B} T\right)^{4}$ and of lower orders in the electromagnetic Casimir free energy (see (44) and (47) in [24]). This results in the contribution to the force acting on a piston which increases with the increase of the temperature, depends on $\hbar$ and $c$ and does not depend on the position of a piston. The same results are obtained from the application of (32). This is because the contribution of the blackbody radiation to the energy of entire box is equal to $-a b c f_{\mathrm{bb}}$ and does not depend on the position of a piston. The contributions from the terms proportional to the surface areas and the sums of the side lengths for the two boxes to the left and to the right of a piston are also independent of the piston position.

\section{Conclusions and discussion}

In the above we have reconsidered the old problem of thermal Casimir force in rectangular boxes made of ideal metal. Our main concern was with the appropriate definition of the Casimir free energy inside some closed volume. We have stressed that such a definition must include a subtraction of the energy of blackbody radiation confined in this volume and of two other terms proportional to $\left(k_{B} T\right)^{3}$ and $\left(k_{B} T\right)^{2}$ which are contained in the high temperature (large separation) asymptotic expression for the thermal correction to the Casimir energy $\Delta_{T} \mathcal{F}_{0}$ in (24). If this is done, the thermal Casimir force turns into zero when the characteristic sizes of the system go to infinity. According to the results of Sect. 3, the suggested expression (32) for the Casimir free energy is applicable to the limiting case of two plane parallel ideal metal planes and leads to the same result as obtained by many authors in the framework of thermal quantum field theory in Matsubara formulation. Both the free energy and the thermal Casimir force were calculated for the massless scalar field and for the electromagnetic field inside ideal metal rectangular boxes. For the scalar field with Dirichlet boundary conditions the thermal Casimir force is always attractive. It was shown that for the electromagnetic field in rectangular boxes the temperature-dependent contribution to Casimir force can be both positive and negative depending on the side lengths. At high temperatures the Casimir free energy is proportional to $k_{B} T$, i.e., the classical limit is achieved, like it holds for two plane parallel planes. The suggested expression for the Casimir free energy leads to the cancellation of all terms of order $\left(k_{B} T\right)^{4},\left(k_{B} T\right)^{3}$ and $\left(k_{B} T\right)^{2}$ at high temperatures (large separations). This is in accordance with what the physical intuition suggests because such terms depend on the Planck constant and, thus, are of quantum origin.

The above results are obtained for rectangular boxes with the Dirichlet boundary conditions (scalar case) or for ideal metal boxes (electromagnetic case). We would like to emphasize that the final resolution of the problems of Casimir repulsion and of the thermal Casimir effect in rectangular boxes requires the inclusion of real material properties of the boundary surfaces. In the case of closed quantization volumes this is a challenging issue which remains to be explored. 


\section{Acknoledgments}

The authors thank M. Bordag for numerous helpful discussions. G.L.K. and V.M.M. are grateful to the Center of Theoretical Studies and the Institute for Theoretical Physics, Leipzig University, for their kind hospitality. This work was supported by Deutsche Forschungsgemeinschaft, Grant No 436 RUS 113/789/0-4.

\section{References}

1. H. B. G. Casimir, Proc. K. Ned. Akad. Wet. 51, 793 (1948)

2. V. M. Mostepanenko, N. N. Trunov, The Casimir Effect and its Applications (Clarendon Press, Oxford, 1997)

3. K. A. Milton, The Casimir Effect (World Scientific, Singapore, 2001)

4. V. M. Mostepanenko, M. Novello, Phys. Rev. D 63, 115003 (2001)

5. E. Fischbach, D. E. Krause, V. M. Mostepanenko, M. Novello, Phys. Rev. D 64, 075010 (2001)

6. R. S. Decca, E. Fischbach, G. L. Klimchitskaya, D. E. Krause, D. López, V. M. Mostepanenko, Phys. Rev. D 68, $116003(2003)$

7. R. S. Decca, D. López, E. Fischbach, G. L. Klimchitskaya, D. E. Krause, V. M. Mostepanenko, Ann. Phys. (N.Y.) 318, 37 (2005)

8. R. S. Decca, D. López, E. Fischbach, G. L. Klimchitskaya, D. E. Krause, V. M. Mostepanenko, Phys. Rev. D 75, $077101(2007)$

9. R. S. Decca, D. López, E. Fischbach, G. L. Klimchitskaya, D. E. Krause, V. M. Mostepanenko, Eur. Phys. J. C 51, $963(2007)$

10. A. A. Saharian, Phys. Rev. D 70, 064026 (2004)

11. A. A. Saharian, Phys. Rev. D 73, 064019 (2006)

12. A. A. Saharian, Phys. Rev. D 74, 124009 (2006)

13. M. Bordag, U. Mohideen, V. M. Mostepanenko, Phys. Rep. 353, 1 (2001)

14. F. Chen, G. L. Klimchitskaya, V. M. Mostepanenko, U. Mohideen, Phys. Rev. Lett. 97, 170402 (2006)

15. F. Chen, G. L. Klimchitskaya, V. M. Mostepanenko, U. Mohideen, Phys. Rev. B 76, 035338 (2007)

16. W. Lukosz, Physica 56, 109 (1971)

17. S. G. Mamayev, N. N. Trunov, Teor. Matem. Fiz. 38, 345 (1979) [Theor. Math. Phys. 38, 228 (1979)]

18. S. G. Mamayev, N. N. Trunov, Izv. Vuzov, Fizika N9, 51 (1979) [Rus. Phys. J. 22, 966 (1979)]

19. J. Ambjørn, S. Wolfram, Ann. Phys. (N.Y.) 147, 1 (1983)

20. D. Deutsch, P. Candelas, Phys. Rev. D 20, 3063 (1979)

21. A. A. Saharian, Eur. Phys. J. C 52, 721 (2007)

22. R. M. Cavalcanti, Phys. Rev. D 69, 065015 (2004)

23. M. P. Hertzberg, R. L. Jaffe, M. Kardar, A. Scardicchio, Phys. Rev. Lett. 95, 250402 (2005)

24. M. P. Hertzberg, R. L. Jaffe, M. Kardar, A. Scardicchio, Phys. Rev. D 76, 045016 (2007)

25. G. Barton, Phys. Rev. D 73, 065018 (2006)

26. V. N. Marachevsky, Phys. Rev. D 75, 085019 (2007)

27. X.-H. Zhai, X.-Z. Li, Phys. Rev. D 76, 047704 (2007)

28. F. C. Santos, A. Tort, Phys. Lett. B 482, 323 (2000)

29. R. Jáuregui, C. Villarreal, S. Hacyan, Ann. Phys. (N.Y.) 321, 2156 (2006)

30. S. C. Lim, L. P. Teo, J. Phys. A: Math. Theor. 40, 11645 (2007)
31. E. Elizalde, S. D. Odintsov, A. Romeo, A. A. Bytsenko, S. Zerbini, Zeta Regularization Techniques with Applications (World Sci., Singapore, 1994)

32. E. Elizalde, J. Phys. A: Math. Gen. 34, 3025 (2001)

33. E. Elizalde, J. Comp. Appl. Math. 118, 125 (2000)

34. L. S. Brown, G. J. Maclay, Phys. Rev. 184, 1272 (1969)

35. E. C. Titchmarsh, Introduction to the Theory of Fourier Integrals (Oxford Univ. Press, Oxford, 1948)

36. J. S. Dowker, G. Kennedy, J. Phys. A: Math. Gen. 11, 895 (1978)

37. J. Schwinger, L. L. DeRaad, K. A. Milton, Ann. Phys. (N.Y.) 115, 1 (1978)

38. J. Feinberg, A. Mann, M. Revzen, Ann. Phys. (N.Y.) 288, 103 (2001)

39. A. Scardicchio, R. L. Jaffe, Nucl. Phys. B 743, 249 (2006)

40. A. A. Actor, Fortschr. d. Phys. 43, 141 (1995)

41. A. Edery, Phys. Rev. D 75, 105012 (2007)

42. D. V. Vassilevich, Phys. Rep. 388, 279 (2003)

43. V. V. Nesterenko, I. G. Pirozhenko, J. Dittrich, Class. Quant. Grav. 20, 431 (2003) 

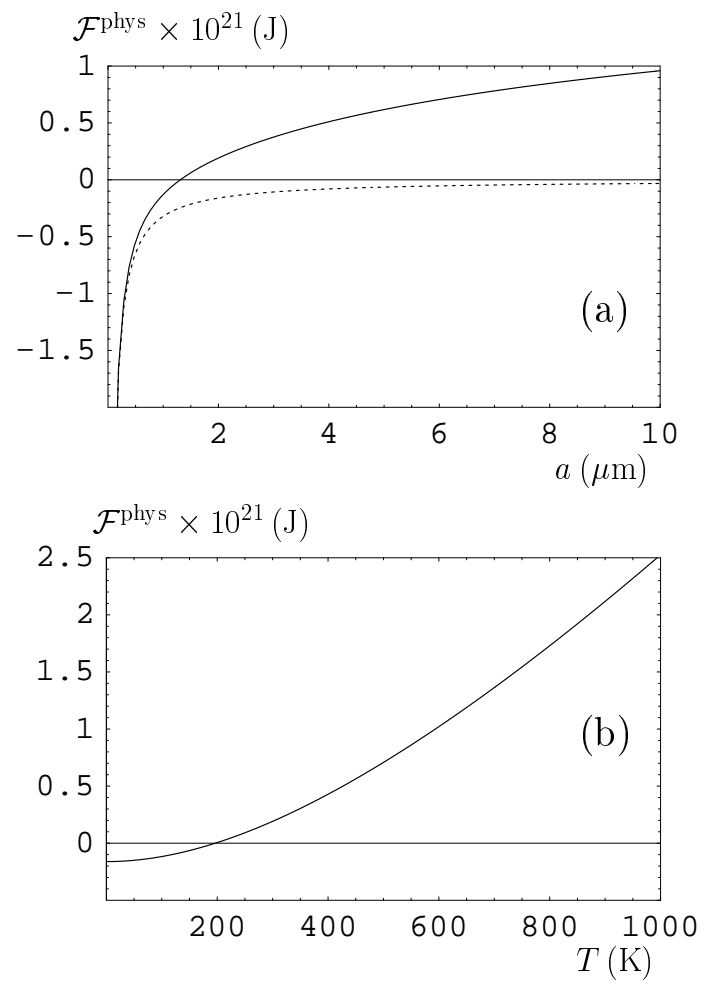

Fig. 1. The scalar Casimir free energy for a cube as a function of (a) a side length $a$ at $T=300 \mathrm{~K}$ (solid line; the dashed line shows the energy at $T=0$ ) and (b) temperature at $a=2 \mu \mathrm{m}$. 

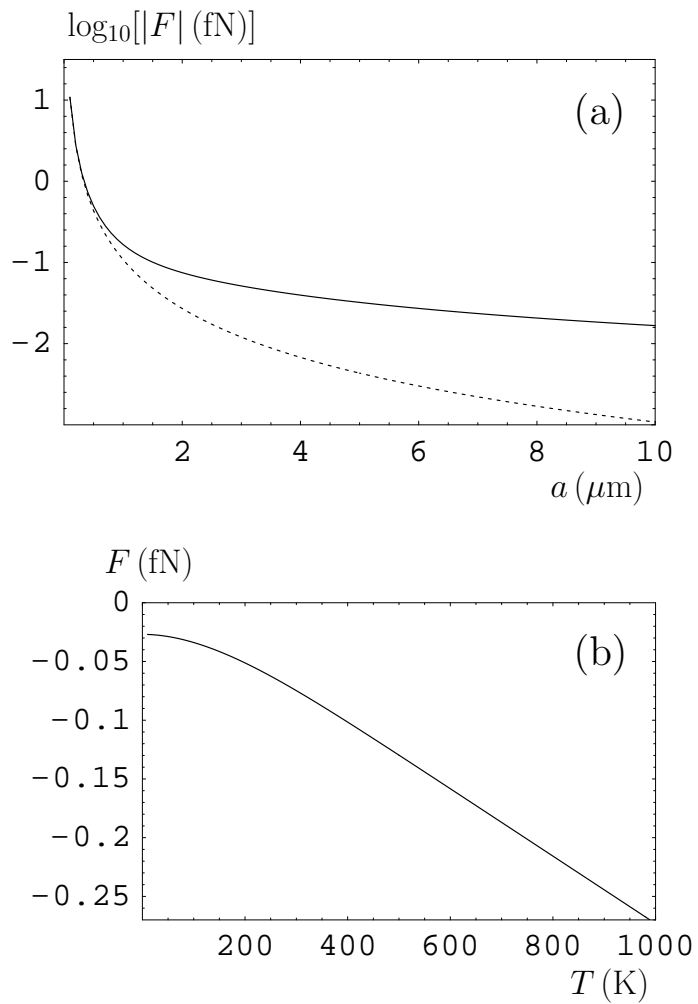

Fig. 2. The scalar Casimir force between the opposite faces of a cube as a function of (a) a side length $a$ at $T=300 \mathrm{~K}$ (solid line; the dashed line shows the force at $T=0)$ and (b) temperature at $a=2 \mu \mathrm{m}$. 

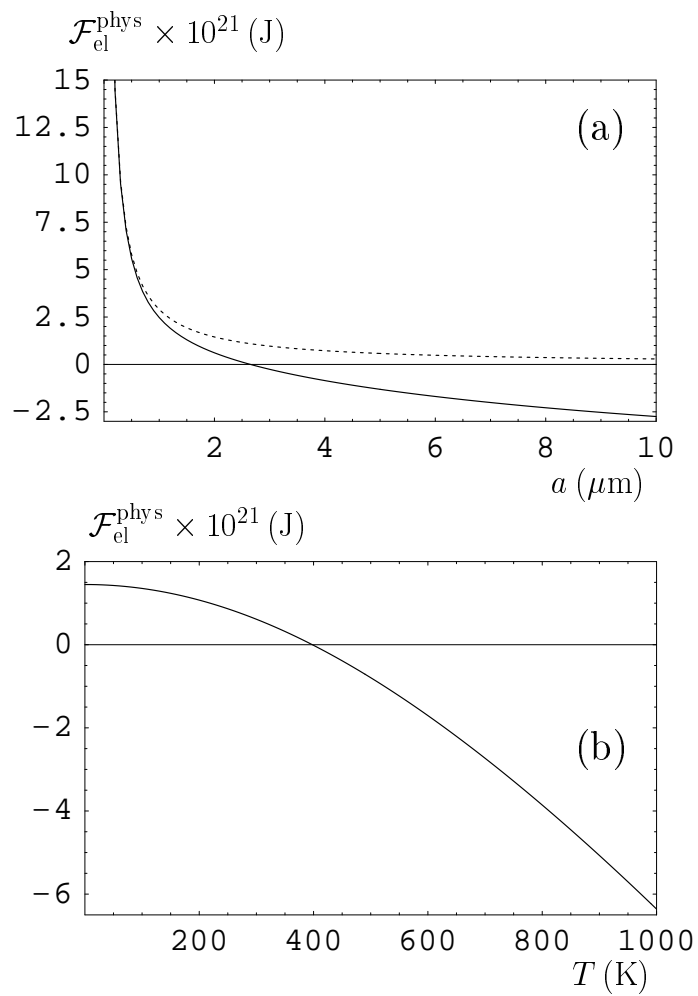

Fig. 3. The electromagnetic Casimir free energy for a cube as a function of (a) a side length $a$ at $T=300 \mathrm{~K}$ (solid line; the dashed line shows the energy at $T=0$ ) and (b) temperature at $a=2 \mu \mathrm{m}$. 

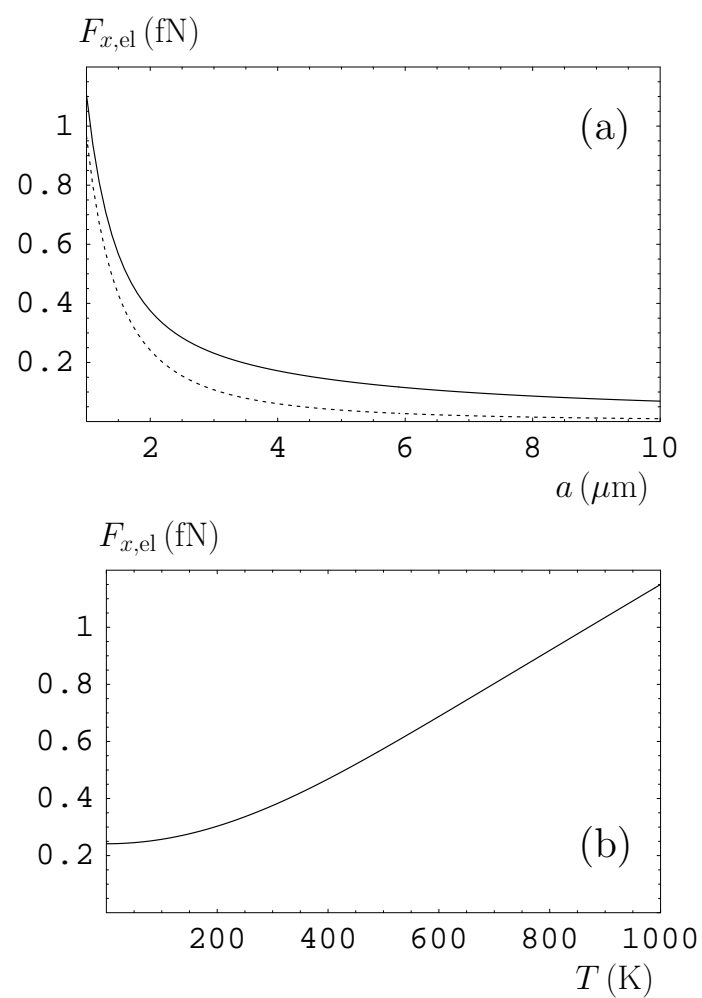

Fig. 4. The electromagnetic Casimir force between the opposite faces of a cube as a function of (a) a side length $a$ at $T=300 \mathrm{~K}$ (solid line; the dashed line shows the force at $T=0$ ) and (b) temperature at $a=2 \mu \mathrm{m}$. 